\title{
A study of friction vibration absorber: impact of friction modeling on the efficacy of the absorber and friction coefficient optimization
}

\author{
Aymen Nasr ${ }^{*}$, Charfeddine Mrad, and Rachid Nasri \\ Laboratory of Applied Mechanics and Engineering (LMAI), National Engineering School of Tunis (ENIT), University of Tunis el \\ Manar (UTM), BP 37, Le Belvedere 1002, Tunis, Tunisia
}

Received: 29 October 2017 / Accepted: 14 October 2018

\begin{abstract}
In this paper, a one degree of freedom system connected to a friction vibration absorber is considered. Friction vibration absorber reduces mechanical vibrations using the dissipation of the energy through friction between both bodies. However, the contact between both bodies and the optimal parameters design deserve to be examined closely. In this paper, the effect of friction modeling is first investigated. Two friction modelings are considered: macroscopic (Coulomb) and microscopic (Dahl and LuGre), according to physical and tribological behaviors. For each model, the responses are determined in sticking and sliding regimes of motion and a comparison is made to conclude on the efficacy of the friction absorber. Second, the optimization of friction coefficient is established using two approaches: Den Hartog optimal parameters and the minimization of the frequency displacement of the main mass.
\end{abstract}

Keywords: Mechanical vibration / vibration reduction / friction absorber / friction modeling / parameters optimization

\section{Introduction}

Dynamic mechanical vibration absorbers are widely used as passive devices to control the level of vibration. Several studies have been developed to improve their effectiveness to reduce mechanical vibrations $[1,2]$. In order to improve the efficiency, a friction element is added to the absorber based on the reliability of friction energy dissipation [3-5]. The friction force, defined as the tangential force between two bodies in contact, is due to various factors like contact geometry and topography, material properties, displacement and speed of bodies, and the presence of a lubricant. Two types of friction of solids are distinguished: macroscopic and microscopic friction [6,7].

Macroscopic modeling assumes that a single dissipative force acts at the interface between the sliding surfaces. This approach is often described as a static friction model. The generalized hysteretic model is one of the most recent models [8], which allows both linear stiffness and friction damping. In this study, the macroscopic model used is the Coulomb model. The Coulomb force takes into account kinetic friction; it is the basic model to represent the force of friction.

\footnotetext{
* e-mail: Aymen.nasr@outlook.com
}

The microscopic modeling is more generalized; it takes into account the details of the sliding surfaces characteristics including roughness, asperities, adhesive phenomena, friction hysteresis, cycle limits, surface lubrication, and other tribological parameters. This approach is often used in the so-called dynamic models [9-11].

Besides, the microscopic modeling represents the stickslip movement in detail. The stick-slip movement manifests itself in the form of repeated sequences of bonding between two surfaces followed by a sliding. In the presence of the friction force, the systems behavior varies from the sticking state to the slipping state, and there is an intermediate state of passage between the two phases. In the sticking phase, Dahl [12] considers the junction points between the contacting surfaces as equivalent to an elastic linkage, which breaks under a tangential loading; he refers to the stress-strain chart of materials. LuGre [13] added the contact junction damping, the Stribeck effect, and the lubrication effect. Mrad et al. [14] studied the effect of friction modeling on the efficiency of the friction absorber to reduce vibration. They used the macroscopic and microscopic approaches to model the friction contact. However, their results are limited to a numerical study and they do not take into account the optimal parameters in consideration. 
After examining the friction modeling, the friction absorber optimization is to be considered. The identification of the optimum parameters leads to the best design of the absorber and increases its efficiency for industrial applications. The optimization consists of finding the optimum parameters of the absorber as a function of the main mass parameters. The researchers proposed several methods to find these parameters. Den Hartog [15] proposed a method for optimizing the parameters of a mass absorber and reducing the sensitivity of the main mass response to the variation of the excitation frequency in a manner that the two resonance peaks of the frequency response are equal. Brock [16] proposed an analytical formulation for the optimum damping rate of the tuned mass damper, found by Den Hartog. Pennestri [17] used a method to minimize the maximum of the frequency amplitude based on an objective function with six equations and seven unknowns. For a system with several degrees of freedom, Abdel-Hafiz and Hassaan [18] have developed an optimization method for a pendulum absorber with three degrees of freedom; they have defined the objective function of optimization by equalizing the responses of the three peaks of resonance.

However, most of the previous optimization studies represent parametric studies constructed from a numerical resolution of the differential equations of motion for mass absorbers. Louroza et al. [19] studied the effect of the mass ratio and the friction force on the frequency response of a vibratory system with a friction absorber. Gewei and Basu [20] studied the effect of the increase of the friction coefficient on the amplitude of the main mass vibration with seismic excitation.

In this article, a friction modeling is elaborated using the macroscopic and microscopic approaches. The system responses are determined analytically for the stick and slip regimes of motion. A comparison of the friction models is made to conclude on the friction absorber efficacy. Then, the optimal friction coefficient is formulated using the Den Hartog parameters and the minimization of the frequency displacement of the main mass.

\section{Vibratory model}

The vibratory system studied, illustrated in Figure 1, is of two degrees of freedom. The main system is modeled by the mass $m_{1}$ and the linear stiffness $k_{1}$. The friction absorber of mass $m_{2}$ is attached to the main system, where $k_{2}$ is the linear stiffness of the absorber.

The contact between the two masses is modeled by the nonlinear friction force $F_{\mathrm{nl}}$; it depends on the normal load $N$ applied by the friction absorber. The main mass is excited by a harmonic force $F_{0} \cos (\Omega t)$, where $F_{0}$ is the amplitude and $\Omega$ is the pulsation.

The motion equations in forced vibration taking into account the friction force are as follows:

$$
\begin{aligned}
& m_{1} \ddot{x}_{1}+\left(k_{1}+k_{2}\right) x_{1}-k_{2} x_{2}+F_{n l}=F_{0} \cos (\Omega t) \\
& m_{2} \ddot{x}_{2}-k_{2}\left(x_{1}-x_{2}\right)-F_{n l}=0 .
\end{aligned}
$$

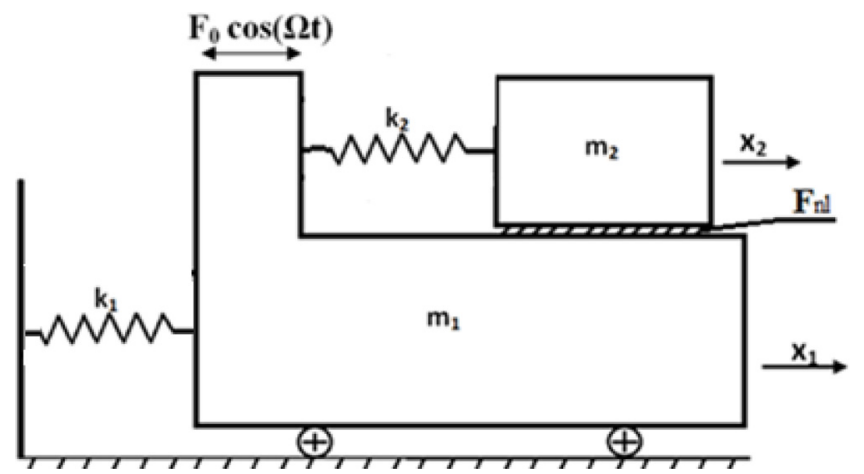

Fig. 1. Main system with friction absorber.

To transform the equations of motion in the sliding case and in the sticking case to the nondimensional form, the following quantities are used:

$$
\begin{aligned}
y_{1} & =\frac{x_{1}}{x_{s}}, y_{2}=\frac{x_{2}}{x_{s}}, x_{s}=\frac{N}{k_{1}}, \omega_{1}=\sqrt{\frac{k_{1}}{m_{1}}}, \omega_{2}=\sqrt{\frac{k_{2}}{m_{2}}}, \omega_{a} \\
& =\frac{\omega_{2}}{\omega_{1}}, r=\frac{m_{2}}{m_{1}}, \tau=\omega_{1} t, \omega=\frac{\Omega}{\omega_{1}}, f_{e}=\frac{F_{0}}{N}
\end{aligned}
$$

The nondimensional equations of motion are then written (Appendix A) as

$$
\begin{aligned}
& \ddot{y}_{1}+\left(1+\omega_{a}^{2} r\right) y_{1}-\omega_{a}^{2} r y_{2}+f_{n l}=f_{e} \cos (\omega \tau) \\
& \ddot{y}_{2}-\omega_{a}^{2}\left(y_{1}-y_{2}\right)-\frac{f_{n l}}{r}=0 .
\end{aligned}
$$

$f_{\mathrm{nl}}$ is the nondimensional form of the friction force $F_{\mathrm{nl}}$; it will be detailed for each model.

The friction force depends on the sign of the relative velocity and on the internal properties of the contact. The diversity of friction model leads to examine friction modeling and determine the steady-state responses. Two modeling approaches, macroscopic and microscopic, and three descriptive models, Coulomb, Dahl, and LuGre, are investigated.

\section{Friction modeling}

\subsection{Macroscopic models}

The friction macroscopic modeling is physical; it supposes a single friction force acting on the contacting surfaces. The Coulomb friction force $F_{\mathrm{nl}}$ as a function of the sign of the relative velocity $v_{r}=\dot{x}_{1}-\dot{x}_{2}$, depends on the normal load and the kinetic friction coefficient. It is written as

$$
F_{n l}=\mu_{c} N \operatorname{sign}\left(\dot{x}_{1}-\dot{x}_{2}\right)
$$

where $N$ is the normal load applied by the mass absorber and $\mu_{\mathrm{c}}$ is the friction coefficient of Coulomb.

Using the nondimensional form, the Coulomb friction force is written as (represented in Fig. 2):

$$
f_{n l}=\mu_{c} \operatorname{sign}\left(\dot{y}_{1}-\dot{y}_{2}\right)
$$




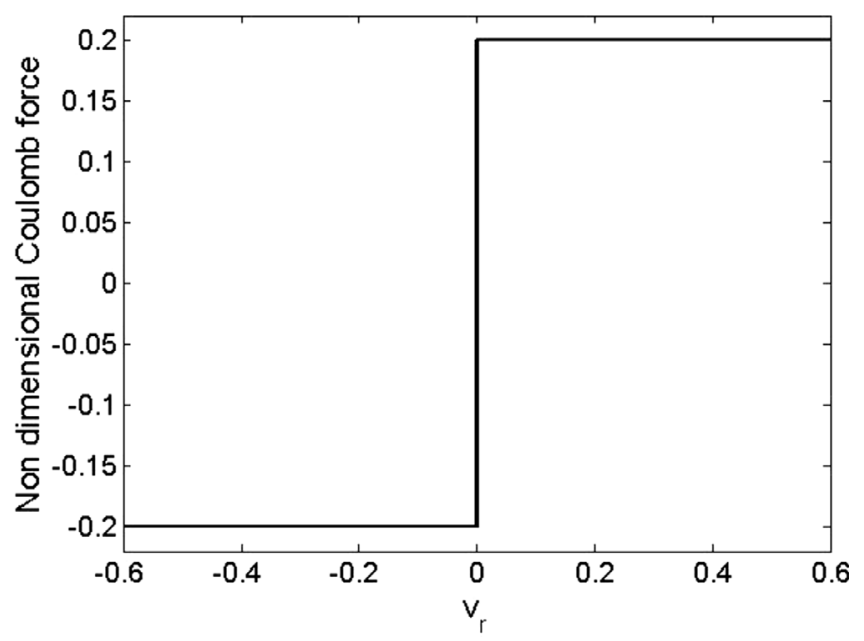

Fig. 2. Coulomb friction force as a function of the relative velocity $v_{\mathrm{r}}$.

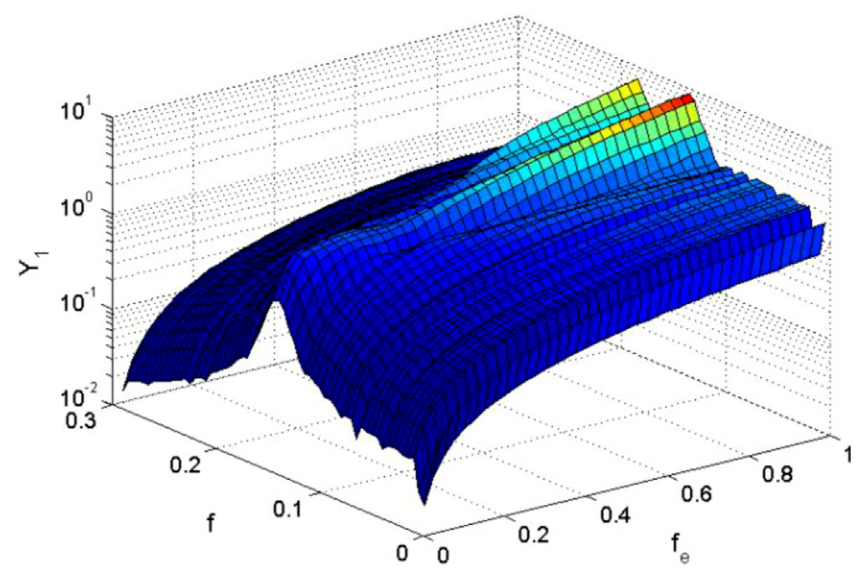

Fig. 3. Main mass displacement $Y_{1}$ as a function of frequency $f$ and amplitude of excitation $f_{e}$, Coulomb.

The system and the absorber are considered undamped. Using the parameters $r=0.1, \omega_{a}=1$, and $\mu_{\mathrm{c}}=0.2$, the system vibratory behavior using Coulomb friction force is investigated.

The friction force as a function of the relative velocity is shown in Figure 2, which shows the stick-slip behavior of the Coulomb friction force. For a nonzero relative velocity, the sign of the friction force is a function of the relative velocity in sliding phase. Additionally, in the sticking phase of motion, the force ratio is defined over an interval between -0.2 and 0.2 .

The stick-slip behavior can be defined as a function of the frequency response $Y_{1}$ of the main mass (Fig. 3). In the sliding phase of motion, the system has two resonant frequencies. While in the sticking phase, the two masses stick together and form one degree of freedom system. This behavior variation depends on the level of the excitation force amplitude; the increase of this force favors the sliding of the two masses, and the system passes from stick motion to the slip motion at the excitation amplitude $f_{\mathrm{e}}=0.36$.

The variation from the stick state to the slip state needs an enough strong excitation force to make a

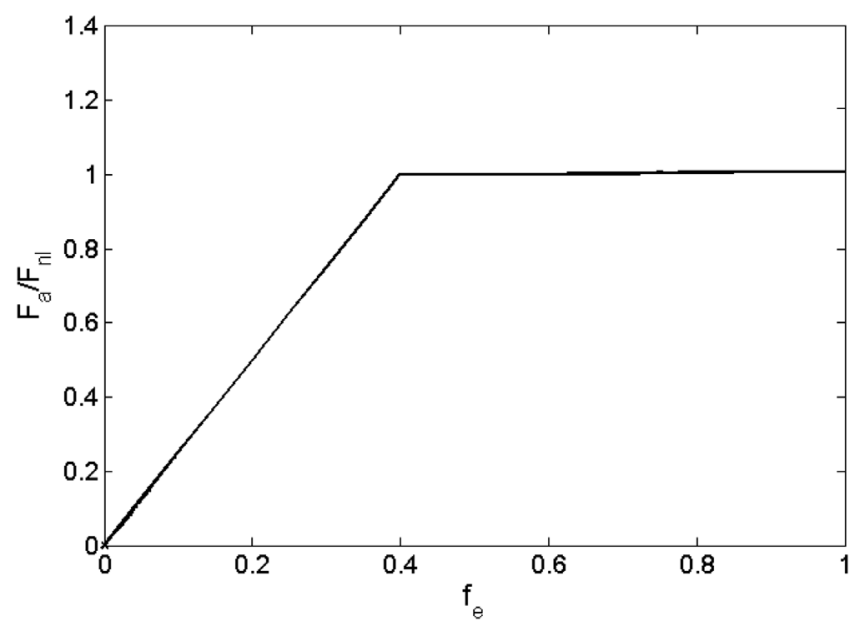

Fig. 4. Forces ratio as a function of excitation force $f_{\mathrm{e}}$, Coulomb.

motion between the two bodies. The difference between the friction force and the maximum inertia force acting on the absorber $F_{a}$ can be calculated by the following equation:

$$
F_{a}=\left|\ddot{y}_{2}-\omega_{a}^{2}\left(y_{1}-y_{2}\right)\right|
$$

Figure 4 shows the change of the absorber acceleration and friction forces ratio for the Coulomb model. To pass from the sticking state to the sliding state, the ratio is equal or superior to 1 ; it means that the maximum inertia force acting on the absorber is superior to the friction force.

The behavior variation affected the responses of the vibratory system. In the sliding zone, the system has two degrees of freedom. The responses can be determined analytically by the equivalent viscous method. The idea of using equivalent damping is to replace the nonlinear friction damping with linear viscous damping that dissipates the same amount of energy during a single vibration cycle.

This amount of energy is defined as

$$
\begin{aligned}
E_{d} & =\int_{\text {cycle }} c_{e}\left(\dot{y}_{1}-\dot{y}_{2}\right) d \tau \\
& =\int_{\text {cycle }} \mu_{c}\left(\dot{y}_{1}-\dot{y}_{2}\right) \operatorname{sign}\left(\dot{y}_{1}-\dot{y}_{2}\right) d \tau .
\end{aligned}
$$

For a harmonic excitation, the solutions sought are harmonic solutions, and $y_{1}(\tau)$ and $y_{2}(\tau)$ are written in the following form

$$
\begin{aligned}
& y_{1}=Y_{1} e^{i \omega \tau} \\
& y_{2}=Y_{2} e^{i \omega \tau}
\end{aligned}
$$

Depending on $Y_{1}$ and $Y_{2}$ and the nondimensional parameters of the system, using equation (6), the equivalent viscous damping rate is defined as

$$
c_{e}=\frac{4 \mu_{c}}{\pi \omega\left(Y_{1}-Y_{2}\right)}
$$


The equivalent viscous damping force is written as

$$
f_{d}=c_{e}\left(\dot{y}_{1}-\dot{y}_{2}\right) \text {. }
$$

Substituting equation (8) of the equivalent viscous damping rate in equation (9) and replacing the nonlinear friction force by the equivalent force in the motion equations (2), the frequency displacement responses $Y_{1}$ and $Y_{2}$ are written as

$Y_{1}=f_{e} \sqrt{\frac{\left(1-\frac{\omega_{a}^{2}}{\omega^{2}}\right)^{2}+\left(\frac{c_{e}}{\omega r}\right)^{2}}{\left(1+(r+1) \omega_{a}^{2}-\omega^{2}-\frac{\omega_{a}^{2}}{\omega^{2}}\right)+c_{e}^{2} \omega^{2}\left(1+\frac{1}{r}-\frac{1}{r \omega^{2}}\right)^{2}}}$

$Y_{2}=f_{e} \sqrt{\frac{\left(\frac{\omega_{a}^{2}}{\omega^{2}}\right)^{2}+\left(\frac{c_{e}}{\omega r}\right)^{2}}{\left(1+(r+1) \omega_{a}^{2}-\omega^{2}-\frac{\omega_{a}^{2}}{\omega^{2}}\right)+c_{e}^{2} \omega^{2}\left(1+\frac{1}{r}-\frac{1}{r \omega^{2}}\right)^{2}}}$

Further, at the sticking phase of motion, the two masses stick together and the system is of one degree of freedom, the differential equation of motion is

$$
\left(m_{1}+m_{2}\right) \ddot{x}_{1}+k_{1} y_{1}=F_{0} \cos (\Omega t) .
$$

The nondimensional form of this equation is written as

$$
\ddot{y}_{1}+\frac{1}{1+r} y_{1}=\frac{f_{e}}{1+r} \cos (\omega \tau) \text {. }
$$

The frequency response using equation (12) is thus written as

$$
Y_{1}=Y_{2}=\frac{f_{e}}{\sqrt{\left(1-\omega^{2}(1+r)\right)^{2}}}
$$

The change from stick to slip motion depends on the kinetic friction coefficient. In other words, the two masses stick if the absorber motion force is less than the kinetic friction force; the condition of sticking is then

$$
\left|\ddot{y}_{2}-\omega_{a}^{2}\left(y_{1}-y_{2}\right)\right| \leq \frac{\mu_{c}}{r}
$$

At this point, the displacements of the two masses are equal and the velocities are also equal. From equations (13) and (14), we obtain the limit friction coefficient as following:

$$
\mu_{\text {limit }}=\sqrt{\frac{f_{e}\left(\omega^{2} r\right)^{2}}{\left(1-\omega^{2}(1+r)\right)^{2}}} \leq \mu_{c}
$$

\subsection{Microscopic models}

The microscopic modeling takes into account the internal properties of the contact. It is a tribological modeling. According to Dahl, the junction between the surfaces of the contact is equivalent to an elastic stiffness, which breaks under tangential force. The Dahl force is defined as

$$
\begin{aligned}
& F_{n l}=\sigma_{0} z, \\
& \dot{z}=\dot{x}_{1}-\dot{x}_{2}-\frac{\sigma_{0}\left|\dot{x}_{1}-\dot{x}_{2}\right|}{\mu_{c} N} z,
\end{aligned}
$$

where $z$ is the internal displacement of the junctions and $\sigma_{0}$ is the contact junction stiffness.

LuGre added the contact junction damping and the Stribeck effect to the Dahl model. In this study, the velocity of Stribeck is taken equal to zero and the static friction coefficient is equal to the kinetic friction coefficient. In this case, the LuGre model equations are written as

$$
\begin{aligned}
& F_{n l}=z \sigma_{0}+\dot{z} \sigma_{1}, \\
& \dot{z}=\dot{x}_{1}-\dot{x}_{2}-\frac{\sigma_{0}}{\mu_{c} N}\left|\dot{x}_{1}-\dot{x}_{2}\right| z .
\end{aligned}
$$

Here $\sigma_{1}$ indicates the viscous damping of the contact junction. If $\sigma_{1}$ is zero, we obtain the Dahl model of elastic contact.

The following quantities are defined to transform equations (15) and (16) to a nondimensional form:

$$
\omega_{3}=\sqrt{\frac{\sigma_{0}}{m_{2}}} \omega_{b}=\frac{\omega_{3}}{\omega_{1}}, \xi_{l}=\frac{\sigma_{1}}{2 m_{2} \omega_{3}}, \text { and } y_{3}=\frac{z}{x_{s}}
$$

The nondimensional Dahl friction model is thus written as follows (Appendix B):

$$
\begin{aligned}
& f_{n l}=r \omega_{b}^{2} y_{3}, \\
& \dot{y}_{3}=\dot{y}_{1}-\dot{y}_{2}-r \frac{\omega_{b}^{2}}{\mu_{c}}\left|\dot{y}_{1}-\dot{y}_{2}\right| y_{3} .
\end{aligned}
$$

The nondimensional LuGre friction model is then written as follows (Appendix B):

$$
\begin{aligned}
& f_{n l}=r \omega_{b}^{2} y_{3}+2 \xi_{l} r \omega_{b} \dot{y}_{3}, \\
& \dot{y}_{3}=\dot{y}_{1}-\dot{y}_{2}-r \frac{\omega_{b}^{2}}{\mu_{c}}\left|\dot{y}_{1}-\dot{y}_{2}\right| y_{3} .
\end{aligned}
$$

Using Dahl and LuGre friction forces, the vibratory system behavior is examined. The problem parameters are the same as used in macroscopic modeling. The internal parameters of friction are, respectively, $\omega_{\mathrm{b}}=0.4$ and $\xi_{1}=0.01$.

Figures 5 and 6 show that two zones exist for each model. For high relative velocity, the friction force takes a value depending on the relative velocity sign; this is similar to the Coulomb friction model. For low relative velocity, the zone of sticking motion is represented with a hysteretic behavior. 


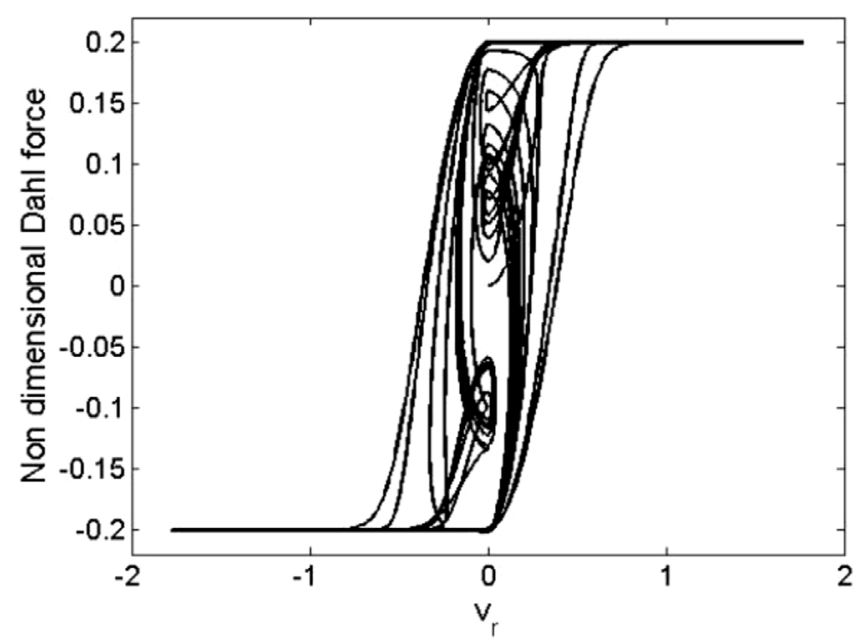

Fig. 5. Dahl friction force as a function of the relative velocity $v_{\mathrm{r}}$.

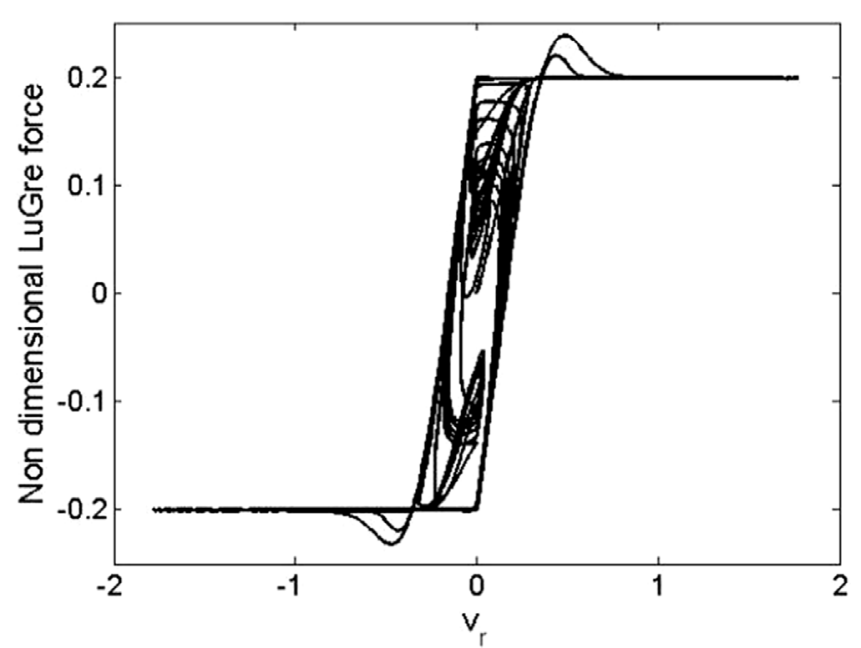

Fig. 6. LuGre friction force as a function of the relative velocity $v_{\mathrm{r}}$.

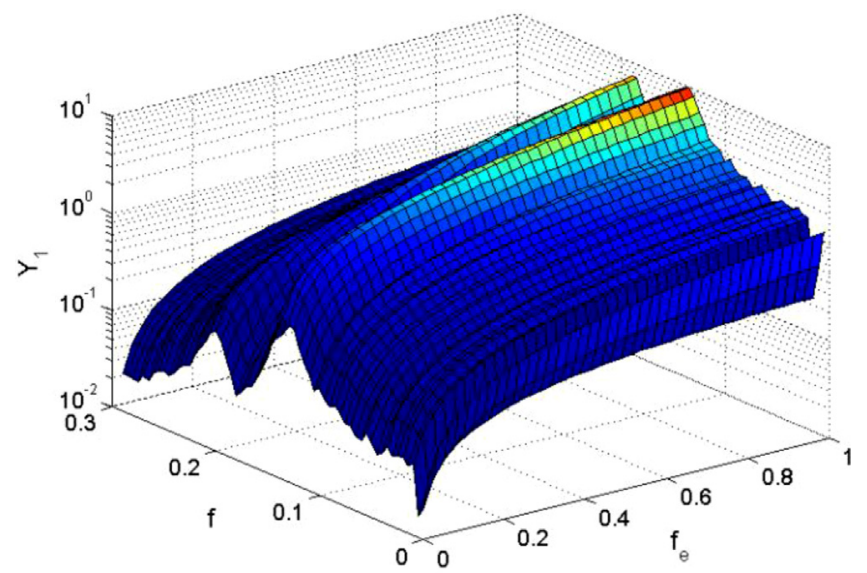

Fig. 7. Main mass displacement $Y_{1}$ as a function of frequency $f$ and amplitude of excitation $f_{\mathrm{e}}$, Dahl.

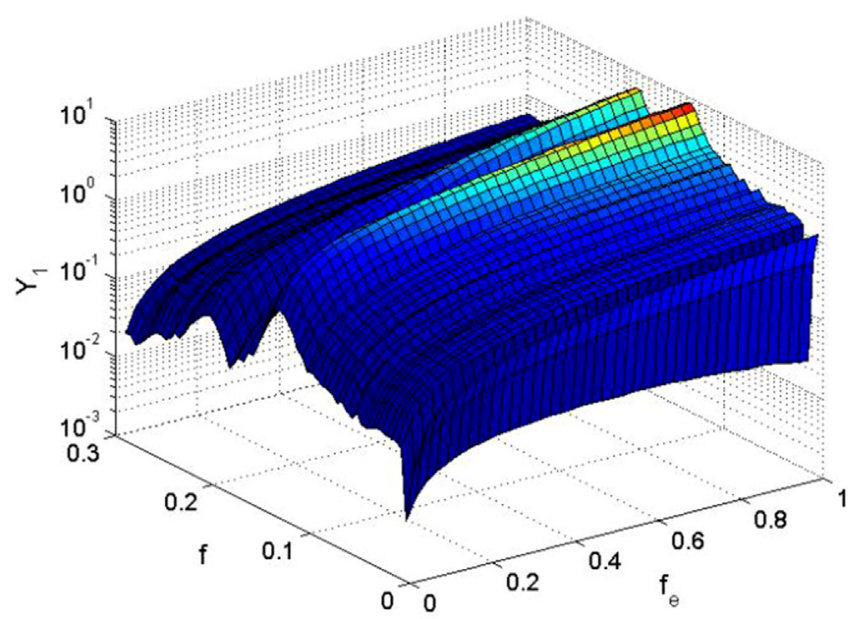

Fig. 8. Main mass displacement $Y_{1}$ as a function of frequency $f$ and amplitude of excitation $f_{\mathrm{e}}$, LuGre.

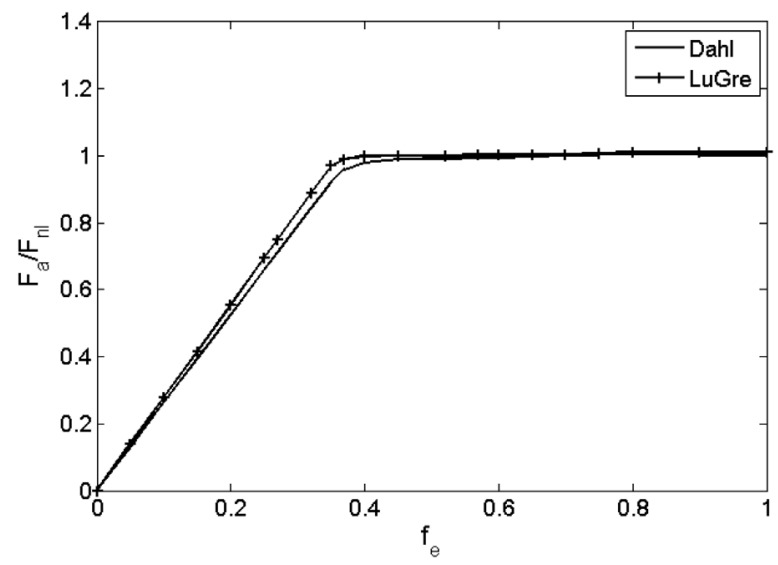

Fig. 9. Forces ratio as a function of excitation force $f_{\mathrm{e}}$, Dahl and LuGre.

For both friction models, the sticking phenomenon occurs when the amplitude of the excitation force is weak (Figs. 7 and 8). The mechanical system has two resonant frequencies; these frequencies are those of a linear two degrees of freedom system in the sticking phase of motion. For the Dahl model, the internal stiffness operates simultaneously with the stiffness of the friction absorber. The friction absorber is similar to an undamped mass absorber. For the LuGre model, the internal damping is added and the friction absorber works as a damped mass absorber. On the other hand, the increase of the amplitude of the excitation force modifies the behavior of the vibratory system.

Using equation (5), Figure 9 shows the evolution of the absorber acceleration and the friction force ratio for Dahl and LuGre frictions. It is clearly seen that the force ratio of LuGre model is higher than that of Dahl model. 


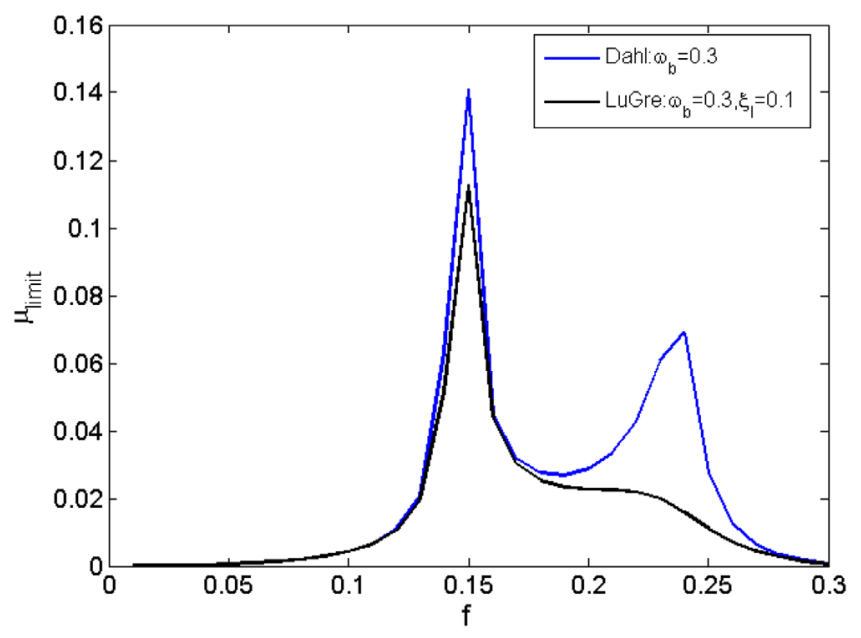

Fig. 10. Limit friction coefficient, microscopic models.

In the sticking phase of motion, the internal stiffness and the internal damping operate. The generalized motion equations in the microscopic modeling are written in the following form:

$$
\begin{aligned}
& m_{1} \ddot{x}_{1}+k_{1} x_{1}+k_{2}\left(x_{1}-x_{2}\right)+\sigma_{1}\left(\dot{x}_{1}-\dot{x}_{2}\right) \\
& \quad+\sigma_{0}\left(x_{1}-x_{2}\right)=F_{0} \cos (\Omega t) m_{2} \ddot{x}_{2}-k_{2}\left(x_{1}-x_{2}\right) \\
& \quad-\sigma_{1}\left(\dot{x}_{1}-\dot{x}_{2}\right)-\sigma_{0}\left(x_{1}-x_{2}\right)=0 .
\end{aligned}
$$

The nondimensional form of equation (20) is written as (Appendix C) as follows:

$$
\begin{gathered}
\ddot{y}_{1}+\left(1+\omega_{a}^{2} r\right) y_{1}-\omega_{a}^{2} r y_{2}+2 \xi_{l} r \omega_{b}\left(\dot{y}_{1}-\dot{y}_{2}\right)+r \omega_{b}^{2}\left(y_{1}-y_{2}\right) \\
=f_{e} \cos (\omega \tau), \ddot{y}_{2}-\omega_{a}^{2}\left(y_{1}-y_{2}\right) \\
\quad-2 \xi_{l} \omega_{b}\left(\dot{y}_{1}-\dot{y}_{2}\right)-\omega_{b}^{2}\left(y_{1}-y_{2}\right)=0 .
\end{gathered}
$$

If the internal damping $\xi_{l}$ is equal to zero, these equations represent the system with Dahl friction in the sticking phase of motion.

Substituting the solutions from equation (7) into equation (21), the frequency responses of the main mass and of the absorber are written as follows:

See the equations (22) and (23) below
For the Dahl model, the sticking occurs if the Coulomb friction force is superior to the force created by the internal stiffness. This condition is written as

$$
\left|\omega_{b}^{2}\left(y_{1}-y_{2}\right)\right| \leq \mu_{c}
$$

The condition of sticking can be determined from equations (22)-(24); it is written as

$$
\mu_{\text {limit }}=\frac{r \omega^{2} \omega_{b}^{2}}{\sqrt{\left(\left(1-\omega^{2}\right)\left(\omega_{a}^{2}+\omega_{b}^{2}-\omega^{2}\right)-r \omega^{2}\left(\omega_{a}^{2}+\omega_{b}^{2}\right)\right)^{2} \leq \mu_{c}}}
$$

However, considering the internal damping, the sticking occurs if the Coulomb friction force is superior to the force created by the internal stiffness and the damping of LuGre model:

$$
\left|\omega_{b}^{2}\left(y_{1}-y_{2}\right)+2 \xi_{l} \omega_{b}\left(\dot{y}_{1}-\dot{y}_{2}\right)\right| \leq \mu_{c} .
$$

Using equations (22), (23), and (26), the condition of sticking can be determined by the following equation:

See the equation (27) below

The limit friction coefficient is proportional to the frequency. It is maximal at the frequencies of resonance (Fig. 10). The limit friction coefficient of LuGre model is inferior to the one of Dahl model.

This coefficient tends to the limit friction coefficient of Coulomb with the increase of the internal stiffness ratio. The augmentation of the internal damping rate favors the decrease of the limit friction coefficient (Fig. 11).

\subsection{Comparison}

The purpose of the comparison is to determine the most efficient model to reduce the vibration of the mechanical system, based on friction modeling.

All macroscopic and microscopic models are based on Coulomb modeling. Since the difference between kinematic and static friction is low, the Coulomb model is capable of representing friction. Using this model, the system

$$
\begin{aligned}
& Y_{1}=\frac{\sqrt{\left(\omega_{a}^{2}+\omega_{b}^{2}-\omega^{2}\right)^{2}+4 \xi_{l}^{2} \omega_{b}^{2} \omega^{2}}}{\sqrt{\left(\left(1-\omega^{2}\right)\left(\omega_{a}^{2}+\omega_{b}^{2}-\omega^{2}\right)-r \omega^{2}\left(\omega_{a}^{2}+\omega_{b}^{2}\right)\right)^{2}+4 \xi_{l}^{2} \omega_{b}^{2} \omega^{2}\left(1-\omega^{2}(1+r)\right)^{2}}}, \\
& Y_{2}=\frac{\sqrt{\left(\omega_{a}^{2}+\omega_{b}^{2}\right)^{2}+4 \xi_{l}^{2} \omega_{b}^{2} \omega^{2}}}{\sqrt{\left(\left(1-\omega^{2}\right)\left(\omega_{a}^{2}+\omega_{b}^{2}-\omega^{2}\right)-r \omega^{2}\left(\omega_{a}^{2}+\omega_{b}^{2}\right)\right)^{2}+4 \xi_{l}^{2} \omega_{b}^{2} \omega^{2}\left(1-\omega^{2}(1+r)\right)^{2}}} . \\
& \mu_{\text {limit }}=\frac{r \omega^{2} \sqrt{\omega_{b}^{4}+4 \xi_{l}^{2} \omega_{b}^{2} \omega^{2}}}{\sqrt{\left(\left(1-\omega^{2}\right)\left(\omega_{a}^{2}+\omega_{b}^{2}-\omega^{2}\right)-r \omega^{2}\left(\omega_{a}^{2}+\omega_{b}^{2}\right)\right)^{2}+4 \xi_{l}^{2} \omega_{b}^{2} \omega^{2}\left(1-\omega^{2}(1+r)\right)^{2}}} \leq \mu_{c .}
\end{aligned}
$$




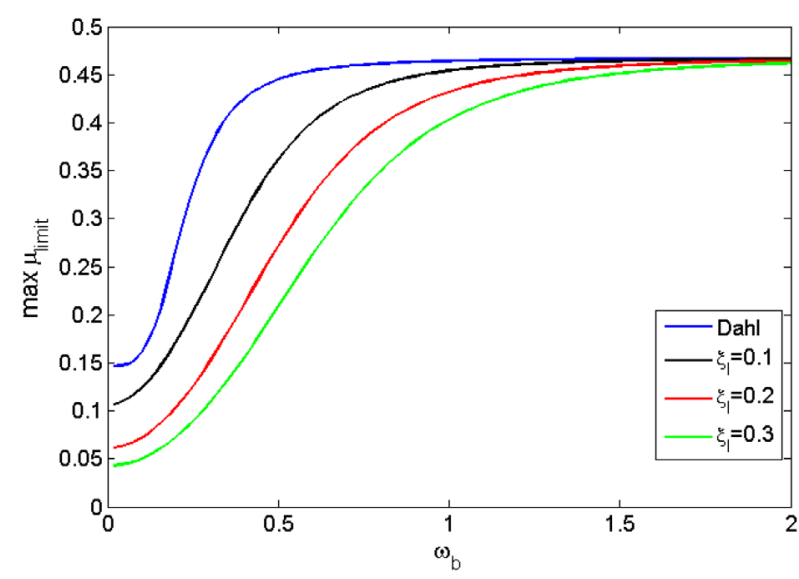

Fig. 11. Maximum limit friction coefficient.

balances between two degrees of freedom system in the slip regime of motion, and a one degree of freedom system in the stick regime of motion.

Microscopic models, Dahl and LuGre, are able to explore the internal properties of the contact. These two models are identical to the Coulomb model in the slip regime. The Dahl model has elastic contact, whereby the friction absorber is equivalent to an undamped mass absorber in the sticking regime of motion. By adding the damping to the contact, the LuGre model presents a viscoelastic contact. In the sticking regime of motion, the friction absorber is equivalent to a damped mass absorber.

All models are similar to Coulomb in the slipping regime of motion. The difference occurs in the sticking regime of motion. In terms of vibratory response, a friction absorber with a viscoelastic friction contact (LuGre model) is more effective in reducing mechanical vibrations.

In the following, we proceed to the optimization of the friction coefficient based on Den Hartog results, and then use the minimization of frequency displacement response.

\section{Parameters optimization}

The optimization consists of determining the optimum friction coefficient to reduce significantly the vibration amplitude. The first method uses Den Hartog approach to find the optimal friction coefficient; the friction absorber is similar to a damped mass absorber. The second method uses Ricciardelli and Vickery approach, the minimum amplitude of vibration of the main mass corresponds to the limit friction coefficient.

\subsection{Den Hartog optimization parameters}

The optimization starts with the determination of the parameters of the friction absorber at the slipping regime. In this case, the friction force is equivalent to a viscous damping force. Using Den Hartog optimal parameters [15], the optimum tuning parameter is given by

$$
\omega_{a o p}=\frac{1}{1+r} \text {. }
$$

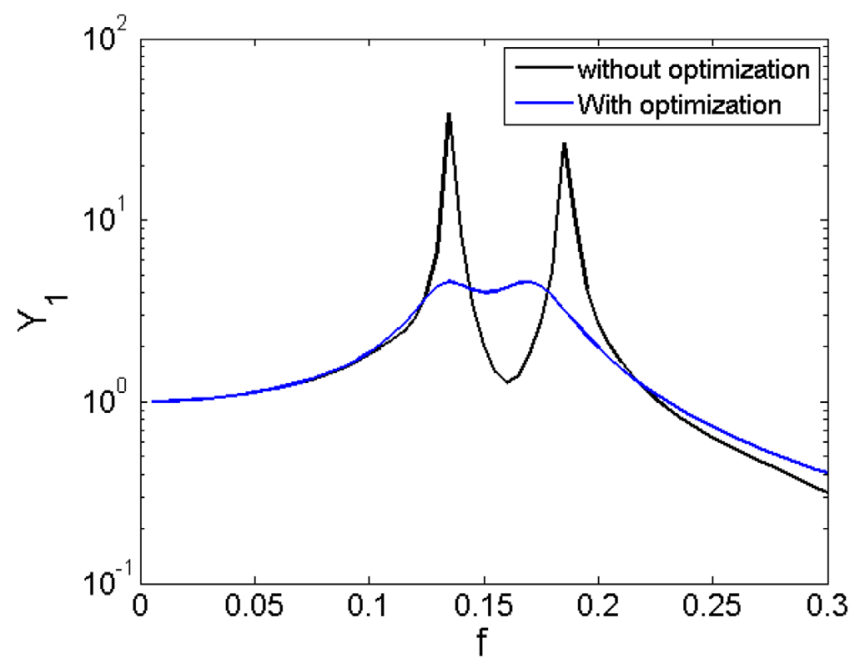

Fig. 12. Frequency responses of the main mass without and with optimization, $r=0.1$.

In addition, the optimum damping rate for the absorber is given by

$$
\xi_{o p}=\sqrt{\frac{3 r}{8(1+r)}} .
$$

To determine the optimal friction coefficient, we express the equivalent viscous damping as a function of a viscous damping ratio $\xi_{\mathrm{e}}($ Appendix D):

$$
c_{e}=2 \xi_{e} \omega_{a} r
$$

Equating equations (8) and (30), we obtain the equivalent viscous damping ratio as

$$
\xi_{e}=\frac{2 \mu}{\pi \omega r \omega_{a}\left(Y_{1}-Y_{2}\right)} .
$$

In order to determine the optimal coefficient, the equivalent viscous damping $\xi_{\mathrm{e}}$ must be equal to the optimal damping $\xi_{\text {op }}$. The optimal friction coefficient is thus defined as

$$
\mu_{o p}=\frac{3 r\left(\pi f_{e} \omega \frac{r}{1+r}\right)^{2}}{32(1+r)\left(2-\omega^{2}-\frac{1}{\omega^{2}}\right)^{2}+48 r \omega^{2}\left(1-\frac{1}{(1+r) \omega^{2}}\right)^{2}} .
$$

Figure 12 shows the effect of the optimal parameters on the response of the main mass with a mass ratio $r=0.1$, as an example. It is clear that the two obtained peaks (with optimization) are equal and the maximum displacement with optimization is less than the maximum displacement without optimization.

The friction coefficient depends on the mass ratio and on the excitation frequency (Fig. 13). The augmentation of the mass ratio favors the increase of the optimal friction coefficient. At the resonance, the friction coefficient has the 


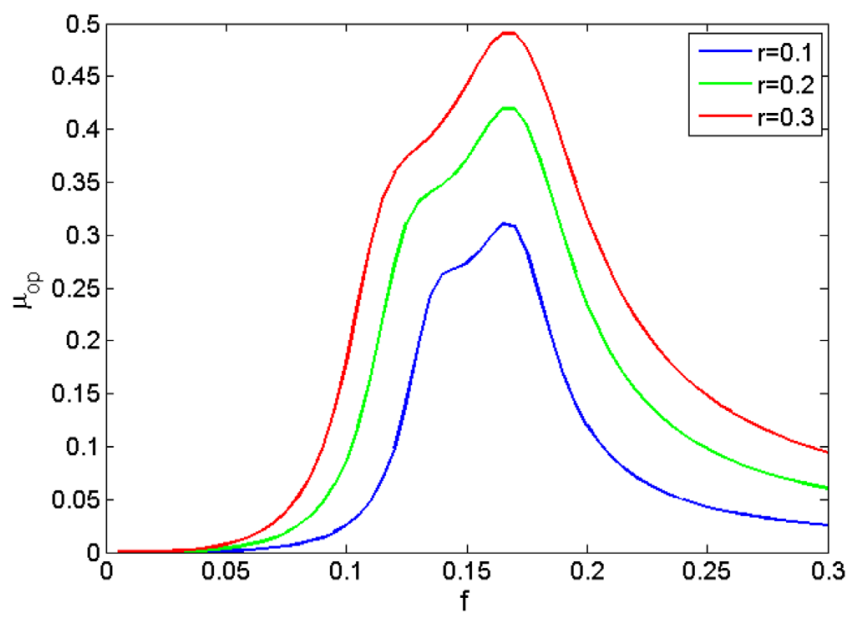

Fig. 13. Optimal friction coefficients.

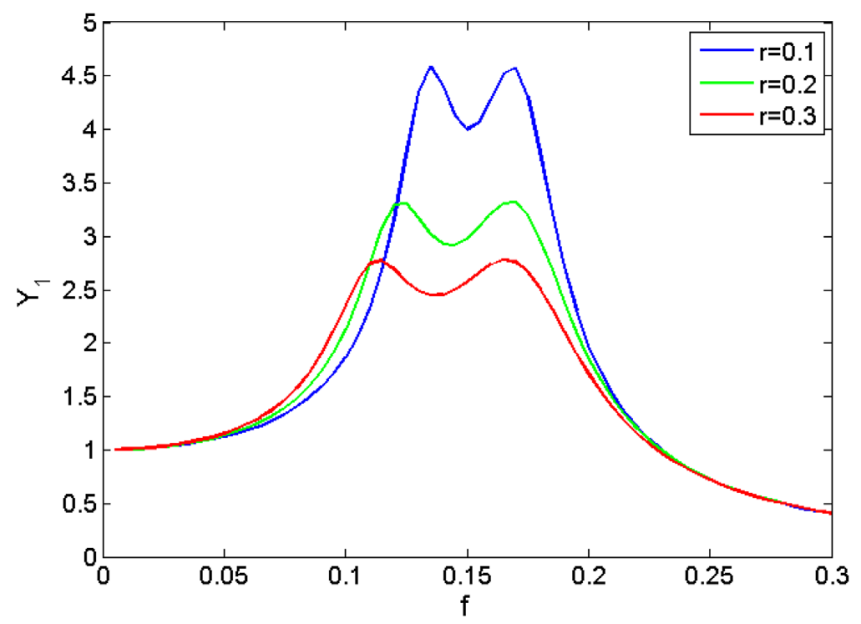

Fig. 14. Optimal frequency responses.

maximum value. This value corresponds to the maximum displacement response of the main mass (Fig. 14).

The variation of the optimal friction coefficient is a function of the excitation frequency. In other words, for each frequency there is an optimal friction coefficient. However, this method of optimization does not take into account the internal parameters of friction.

\subsection{Minimization of frequency displacement}

According to Ricciardelli and Vickery [21], the minimum amplitude of vibration of the main mass corresponds to the limit friction coefficient, which separates the two zones of motion (sticking and slipping). From equation (22), the optimal friction coefficient is written as

See the equation (33) below

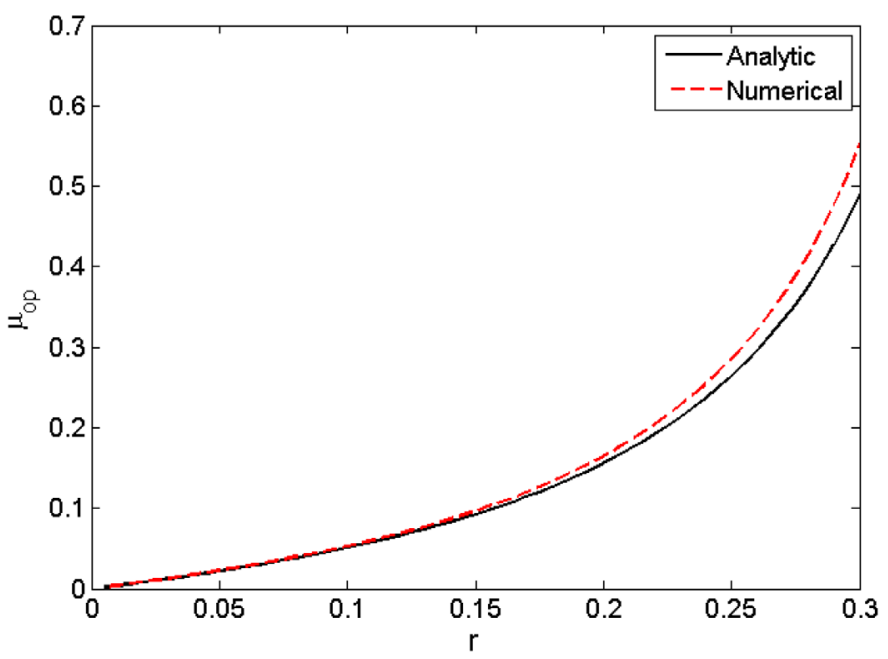

Fig. 15. Optimal friction coefficient at $f=0.12 ; r=0.1, \omega_{\mathrm{a}}=1$, $\omega_{\mathrm{b}}=0.4$, and $\xi_{1}=0.1$.

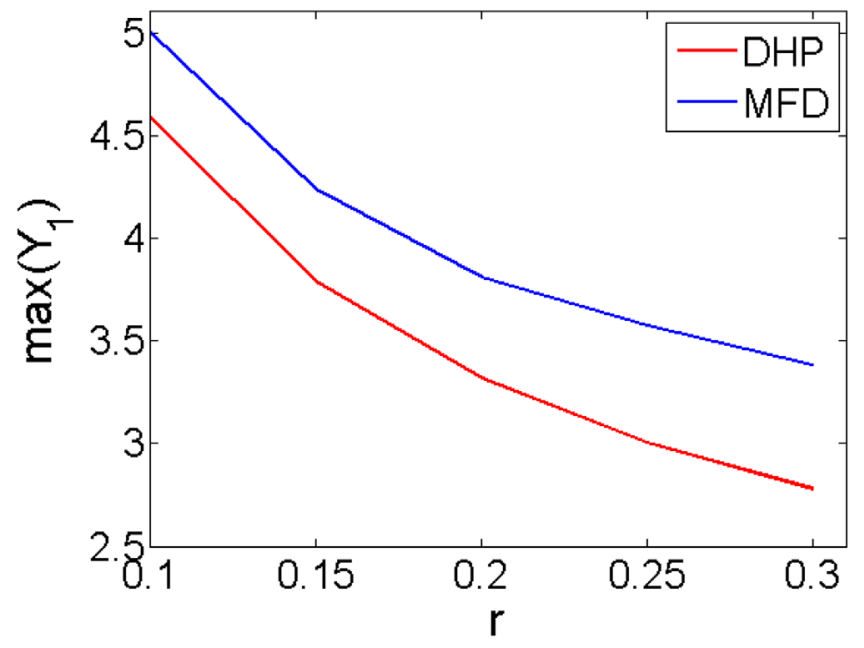

Fig. 16. Optimal maximum displacement $Y_{1}$.

This coefficient depends on the main mass and the absorber parameters. For each frequency, there exists an optimal friction coefficient. Figure 15 shows the coincidence of the numerical and analytical optimal friction coefficients at $f=0.12$, as an example.

Regarding the maximum displacement of the main mass at resonance obtained by the two methods of optimization (Fig. 16), the first method (Den Hartog parameters) is more effective in reducing vibration than the second method (minimization of frequency displacement).

$$
\mu_{o p}=\frac{r \omega^{2} \sqrt{\omega_{b}^{4}+4 \xi_{l}^{2} \omega_{b}^{2} \omega^{2}}}{\sqrt{\left(\left(1-\omega^{2}\right)\left(\omega_{a}^{2}+\omega_{b}^{2}-\omega^{2}\right)-r \omega^{2}\left(\omega_{a}^{2}+\omega_{b}^{2}\right)\right)^{2}+4 \xi_{l}^{2} \omega_{b}^{2} \omega^{2}\left(1-\omega^{2}(1+r)\right)^{2}}}
$$




\section{Conclusion}

To examine the friction modeling, the macroscopic and the microscopic modeling approaches are considered. The macroscopic modeling is based on the Coulomb friction, characterized by the kinetic friction. The microscopic modeling is investigated using the elastic contact (Dahl) and the viscoelastic contact (LuGre). For each friction model, an analytic study is elaborated to determine the responses at the sticking and the sliding regimes of motion. For each friction model, there is limit friction coefficient between the regimes of motion. The comparison of the different friction models shows that the LuGre model is the most effective in reducing vibrations.

The friction absorber parameters optimization is elaborated using two methods. The Den Hartog optimal parameters theory cannot take into account the internal parameters of friction models. The minimization of frequency displacement method of the main mass is the most generalized. However, the obtained displacement using the first method is less than the one obtained by the second method. The comparison of the two methods leads to conclude that the damped mass absorber may be more effective than the friction absorber.

\section{Appendix A}

Defining the nondimensional time as $\tau=\omega_{1} t$ and dividing equation (1) by $m_{1}$ for the first, and by $m_{2}$ for the second, the equations of motion are expressed as follows:

$$
\begin{aligned}
& \omega_{1}^{2} \ddot{x}_{1}+\frac{\left(k_{1}+k_{2}\right)}{m_{1}} x_{1}-\frac{k_{2}}{m_{1}} x_{2}+\frac{F_{n l}}{m_{1}}=\frac{F_{0}}{m_{1}} \cos (\omega \tau), \\
& \omega_{1}^{2} \ddot{x}_{2}-\frac{k_{2}}{m_{2}}\left(x_{1}-x_{2}\right)-\frac{F_{n l}}{m_{2}}=0 .
\end{aligned}
$$

Here $\omega=\frac{\Omega}{\omega_{1}}, \omega_{1}^{2} \ddot{x}_{1}=\omega_{1}^{2} \frac{d^{2} x_{1}(\tau)}{d \tau^{2}}=\frac{d^{2} x_{1}(t)}{d t^{2}}$, and

$\omega_{1}^{2} \ddot{x}_{2}=\omega_{1}^{2} \frac{d^{2} x_{2}(\tau)}{d \tau^{2}}=\frac{d^{2} x_{2}(t)}{d t^{2}}$.

Then, dividing equation (A.1) by $\omega_{1}^{2}$, we obtain

$$
\begin{aligned}
& \ddot{x}_{1}+\left(1+r \omega_{a}^{2}\right) x_{1}-r \omega_{a}^{2} x_{2}+\frac{F_{n l}}{m_{1} \omega_{1}^{2}}=\frac{F_{0}}{m_{1} \omega_{1}^{2}} \cos (\omega \tau), \\
& \ddot{x}_{2}-\omega_{a}^{2}\left(x_{1}-x_{2}\right)-\frac{F_{n l}}{m_{2}}=0 . \\
& \text { Here } r=\frac{m_{2}}{m_{1}}, \omega_{a}^{2}=\frac{\frac{k_{2}}{m_{2}}}{\frac{k_{1}}{m_{1}}}
\end{aligned}
$$

Now, dividing equation (A.2) by $x_{\mathrm{s}}=N / k_{1}$, we obtain

$$
\begin{aligned}
& \ddot{y}_{1}+\left(1+r \omega_{a}^{2}\right) y_{1}-r \omega_{a}^{2} y_{2}+\frac{F_{n l}}{x_{s} m_{1} \omega_{1}^{2}}=\frac{F_{0}}{x_{s} m_{1} \omega_{1}^{2}} \cos (\omega \tau), \\
& \ddot{y}_{2}-\omega_{a}^{2}\left(y_{1}-y_{2}\right)-\frac{F_{n l}}{x_{s} m_{2} \omega_{1}^{2}}=0 .
\end{aligned}
$$

Here $y_{1}=\frac{x_{1}}{x_{s}}, y_{2}=\frac{x_{2}}{x_{s}}, \ddot{y}_{1}=\frac{d^{2}\left(x_{1} / x_{s}\right)}{d \tau^{2}}=\frac{d^{2} x_{1}}{x_{s} d \tau^{2}}=\frac{d \dot{x}_{1}}{x_{s} d \tau}$,

$\ddot{y}_{2}=\frac{d^{2}\left(x_{2} / x_{s}\right)}{d \tau^{2}}=\frac{d^{2} x_{2}}{x_{s} d \tau^{2}}=\frac{d \dot{x}_{2}}{x_{s} d \tau}$.

The nondimensional forces $f_{\mathrm{nl}}$ and $f_{\mathrm{e}}$ are written as follows:

$$
\begin{gathered}
f_{n l}=\frac{F_{n l}}{N}, \\
f_{e}=\frac{F_{0}}{N} .
\end{gathered}
$$

Finally, the equations of motion are defined as

$$
\begin{aligned}
& \ddot{y}_{1}+\left(1+r \omega_{a}^{2}\right) y_{1}-r \omega_{a}^{2} y_{2}+f_{n l}=f_{e} \cos (\omega \tau), \\
& \ddot{y}_{2}-\omega_{a}^{2}\left(y_{1}-y_{2}\right)-\frac{f_{n l}}{r}=0 .
\end{aligned}
$$

\section{Appendix B}

The equations of the microscopic friction force are as follows:

$$
\begin{aligned}
& F_{n l}=z \sigma_{0}+\dot{z} \sigma_{1}, \\
& \dot{z}=\dot{x}_{1}-\dot{x}_{2}-\frac{\sigma_{0}}{\mu_{c} N}\left|\dot{x}_{1}-\dot{x}_{2}\right| z .
\end{aligned}
$$

Dividing the first equation of (B.1) by $x_{s} m_{1} \omega_{1}^{2}$ and using the nondimensional time $\tau=\omega_{1} t$, we obtain

$$
\frac{F_{n l}}{x_{s} m_{1} \omega_{1}^{2}}=\frac{z \sigma_{0}}{x_{s} m_{1} \omega_{1}^{2}}+\frac{\dot{z} \omega_{1} \sigma_{1}}{x_{s} m_{1} \omega_{1}^{2}},
$$

where $\omega_{1} \dot{z}=\omega_{1} \frac{d z(\tau)}{d \tau}=\frac{d z(t)}{d t}$.

The first term is $\frac{F_{n l}}{x_{s} m_{1} \omega_{1}^{2}}=\frac{F_{n l}}{N}=f_{n l}$.

The second term is $\frac{z \sigma_{0}}{x_{s} m_{1} \omega_{1}^{2}}=\frac{z}{x_{s}} \frac{\sigma_{0}}{m_{1} \omega_{1}^{2}} \frac{m_{2}}{m_{2}}=y_{3} r \omega_{b}^{2}$,

where $y_{3}=\frac{z}{x_{s}}, \omega_{b}^{2}=\frac{\sigma_{0}}{m_{2} \omega_{1}^{2}}$

The third term is $\frac{\dot{z} \omega_{1} \sigma_{1}}{x_{s} m_{1} \omega_{1}^{2}}=\frac{\dot{z}}{x_{s}} \frac{\sigma_{1}}{m_{1} \omega_{1}} \frac{m_{2} \omega_{3}}{m_{2} \omega_{3}}=\dot{y}_{3} 2 r \omega_{b} \xi_{l}$, where $\xi_{l}=\frac{\sigma_{1}}{2 m_{2} \omega_{3}}$.

Now, dividing the second equation of (B.1) by $x_{\mathrm{s}}$ and using the nondimensional time $\tau$, we obtain

$$
\frac{\dot{z}}{x_{s}}=\frac{1}{x_{s}}\left(\dot{x}_{1}-\dot{x}_{2}\right)-\frac{1}{x_{s}} \frac{\sigma_{0}}{\mu_{c} N}\left|\dot{x}_{1}-\dot{x}_{2}\right| z
$$


- The first term is $\frac{\dot{z}}{x_{s}}=\frac{d\left(z / x_{s}\right)}{d \tau}=\frac{d z}{x_{s} d \tau}=\dot{y}_{3}$

- The second is $\frac{1}{x_{s}}\left(\dot{x}_{1}-\dot{x}_{2}\right)=\dot{y}_{1}-\dot{y}_{2}$.

- The third term is $\frac{1}{x_{s}} \frac{\sigma_{0}}{\mu_{c} N}\left|\dot{x}_{1}-\dot{x}_{2}\right| z=\frac{\sigma_{0}}{\mu_{c} N}\left|\dot{y}_{1}-\dot{y}_{2}\right| z$.

Multiplying and dividing by $\mathrm{k}_{1} \mathrm{~m}_{1} \mathrm{~m}_{2}$, this term becomes.

$$
\frac{\sigma_{0}}{\mu_{c} N}\left|\dot{y}_{1}-\dot{y}_{2}\right| z \frac{k_{1}}{k_{1}} \frac{m_{1}}{m_{1}} \frac{m_{2}}{m_{2}}=r \frac{\omega_{b}^{2}}{\mu_{c}}\left|\dot{y}_{1}-\dot{y}_{2}\right| y_{3}
$$

Finally, the nondimensional microscopic friction force

$$
\begin{aligned}
& f_{n l}=r \omega_{b}^{2} y_{3}+2 \xi_{l} r \omega_{b} \dot{y}_{3,} \\
& \dot{y}_{3}=\dot{y}_{1}-\dot{y}_{2}-r \frac{\omega_{b}^{2}}{\mu_{c}}\left|\dot{y}_{1}-\dot{y}_{2}\right| y_{3} .
\end{aligned}
$$

\section{Appendix C}

The equations of motion at the sticking regime are as follows:

$$
\begin{aligned}
& m_{1} \ddot{x}_{1}+k_{1} x_{1}+k_{2}\left(x_{1}-x_{2}\right)+\sigma_{1}\left(\dot{x}_{1}-\dot{x}_{2}\right)+\sigma_{0}\left(x_{1}-x_{2}\right) \\
& \quad=F_{0} \cos (\Omega t), \\
& m_{2} \ddot{x}_{2}-k_{2}\left(x_{1}-x_{2}\right)-\sigma_{1}\left(\dot{x}_{1}-\dot{x}_{2}\right) \\
& -\sigma_{0}\left(x_{1}-x_{2}\right)=0 .
\end{aligned}
$$

Using the nondimensional time and dividing the first equation by $m_{1}$ and the second equation by $m_{2}$, equations (C.1) are now written as follows:

$$
\begin{gathered}
\omega_{1}^{2} \ddot{x}_{1}+\frac{k_{1}}{m_{1}} x_{1}+\frac{k_{2}}{m_{1}}\left(x_{1}-x_{2}\right)+\frac{\sigma_{1}}{m_{1}} \omega_{1}\left(\dot{x}_{1}-\dot{x}_{2}\right) \\
+\frac{\sigma_{0}}{m_{1}}\left(x_{1}-x_{2}\right)=\frac{F_{0}}{m_{1}} \cos (\omega \tau) \\
\omega_{1}^{2} \ddot{x}_{2}-\frac{k_{2}}{m_{2}}\left(x_{1}-x_{2}\right)-\frac{\sigma_{1}}{m_{2}} \omega_{1}\left(\dot{x}_{1}-\dot{x}_{2}\right) \\
-\frac{\sigma_{0}}{m_{2}}\left(x_{1}-x_{2}\right)=0
\end{gathered}
$$

Where

$$
\omega_{1} \dot{x}_{1}=\omega_{1} \frac{d x_{1}(\tau)}{d \tau}=\frac{d x_{1}(t)}{d t}
$$

and is defined as

Dividing equations (C.2) by $\omega_{1}^{2}$, the equations are written as follows:

$$
\begin{gathered}
\ddot{x}_{1}+x_{1}+r \omega_{a}^{2}\left(x_{1}-x_{2}\right)+2 r \omega_{b} \xi_{1}\left(\dot{x}_{1}-\dot{x}_{2}\right) \\
+r \omega_{b}^{2}\left(x_{1}-x_{2}\right)=\frac{F_{0}}{m_{1} \omega_{1}^{2}} \cos (\omega \tau) \\
\ddot{x}_{2}-\omega_{a}^{2}\left(x_{1}-x_{2}\right)-2 \omega_{b} \xi_{1}\left(\dot{x}_{1}-\dot{x}_{2}\right)-\omega_{b}^{2}\left(x_{1}-x_{2}\right)=0
\end{gathered}
$$

Now, multiplying and dividing equations (C.2) by $x_{\mathrm{s}}$, we obtain

$$
\begin{gathered}
\ddot{y}_{1}+y_{1}+r \omega_{a}^{2}\left(y_{1}-y_{2}\right)+2 r \omega_{b} \xi_{l}\left(\dot{y}_{1}-\dot{y}_{2}\right) \\
+r \omega_{b}^{2}\left(y_{1}-y_{2}\right)=f_{e} \cos (\omega \tau), \\
\ddot{y}_{2}-\omega_{a}^{2}\left(y_{1}-y_{2}\right)-2 \omega_{b} \xi_{l}\left(\dot{y}_{1}-\dot{y}_{2}\right)-\omega_{b}^{2}\left(y_{1}-y_{2}\right)=0 .
\end{gathered}
$$

\section{Appendix D}

Substituting the equivalent force (9) into the nondimensional equations of motion (2), we obtain

$$
\begin{aligned}
& \ddot{y}_{1}+y_{1}+r \omega_{a}^{2}\left(y_{1}-y_{2}\right)+c_{e}\left(\dot{y}_{1}-\dot{y}_{2}\right)=f_{e} \cos (\omega \tau) \\
& \ddot{y}_{2}-\omega_{a}^{2}\left(y_{1}-y_{2}\right)-\frac{c_{e}}{r}\left(\dot{y}_{1}-\dot{y}_{2}\right)=0
\end{aligned}
$$

The equivalent viscous damping is defined as

$$
c_{e}=2 r \omega_{a} \xi_{e}
$$

Finally, the equations of motion will be treated as a Den Hartog problem where $\omega_{\mathrm{a}}$ and $\xi_{\mathrm{e}}$ are the tuning ratio parameter and the viscous damping rate, respectively. The equations of motion are as follows:

$$
\begin{gathered}
\ddot{y}_{1}+y_{1}+r \omega_{a}^{2}\left(y_{1}-y_{2}\right)+2 r \omega_{a} \xi_{e}\left(\dot{y}_{1}-\dot{y}_{2}\right)=f_{e} \cos (\omega \tau), \\
\ddot{y}_{2}-\omega_{a}^{2}\left(y_{1}-y_{2}\right)-2 \omega_{a} \xi_{e}\left(\dot{y}_{1}-\dot{y}_{2}\right)=0 .
\end{gathered}
$$

\section{References}

[1] K. Liu, J. Liu, The damped dynamic vibration absorbers: revisited and new result, J. Sound Vib. 284 (2005) 1181-1189

[2] C.L. Lee, Y.T. Chen, L.L. Chung, Y.P. Wang, Optimal design theories and applications of tuned mass dampers, Eng. Struct. 28 (2006) 43-53

$\omega_{1} \dot{x}_{2}=\omega_{1} \frac{d x_{2}(\tau)}{d \tau}=\frac{d x_{2}(t)}{d t}$ friction damper, J. Sound Vib. 278 (2004) 474-479 
[4] M. Wang, T. Zan, Y. Yang, R. Fei, Design and implementation of nonlinear TMD for chatter suppression: an application in turning processes, Int. J. Mach. Tools Manuf. 50 (2010) 74-96

[5] D. Brizard, S. Besset, L. Jézéquel, B. Troclet, Design and test of a friction damper to reduce engine vibrations on a space launcher, Arch. Appl. Mech. 83 (2013) 799-815

[6] S. Andersson, A. Soderberg, S. Bjorklund, Friction models for sliding dry, boundary and mixed lubricated contacts, Tribol. Int. 40 (2007) 580-587

[7] G.J. Stein, Dry friction modeling and simulation in kinematically excited oscillatory systems, J. Sound Vib. 311 (2008) 74-96

[8] N. Bouchaala, Impact des défauts géométriques sur l'amortissement dans les assemblages, THESE, école nationales des ingénieurs de Sfax, janvier 2014.

[9] H. Olsson, K.J. Åström, C. Canudas de Wit, M. Gäfvert, P. Lischinsky, Friction models and friction compensation, Eur. J. Control 4 (1998), 176-195.

[10] E.J. Berger, Friction modeling for dynamic system simulation, Appl. Mech. Rev. 55 (2002) 535-577.

[11] J. Awrejcewiczan, P. Olejnik, Analysis of dynamic systems with various friction laws, Appl. Mech. Rev. 58 (2005) 389-411

[12] D. Chou, Dahl Friction Modeling, Massachusetts Institute of Technology, June 2004.
[13] C. Canudas de Wit, H. Olsson, K.J. Astrom, P. Lischinsky, A new model for control of systems with friction, IEEE Trans. Autom. Control, 40 (1995) 419-425

[14] C. Mrad, K. Chehaibi, A. Nasr, R. Nasri, Modeling effect on the performance of impact and friction vibration absorbers, Mechanics \& Industry 18 (2017) 206

[15] J.P. Den Hartog, Mechanical Vibrations, McGraw-Hill, New York (1956)

[16] J.E. Brock, A note on the damped vibration absorber, J. Appl. Mech. 68 (1946) A-284.

[17] E. Pennestrì, An application of Chebyshev's min-max criterion to the optimal design of a damped dynamic vibration absorber, J. Sound Vib. 217 (1998) 757-765

[18] M.A. Abdel-Hafiz, G.A. Hassaan, Minimax optimization of dynamic pendulum absorbers for a damped primary system, Int. J. Sci. Technol. Res. 3 (2014) 209-215

[19] M.A. Louroza, N. Roitman, C. Magluta, Vibration reduction using passive absorption system with Coulomb damping, Mech. Syst. Signal Process. 19 (2005) 537-549

[20] Z. Gewei, B. Basu, A study on friction-tuned mass damper: harmonic solution and statistical linearization, J. Vib. Control 17 (2011) 721-731

[21] F. Ricciardelli, B. Vickery, Tuned vibration absorbers with dry friction damping, Earthq. Eng. Struct. Dyn. 28 (1999) $707-723$

Cite this article as: A. Nasr, C. Mrad, R. Nasri, A study of friction vibration absorber: impact of friction modeling on the efficacy of the absorber and friction coefficient optimization, Mechanics \& Industry 19, 602 (2018) 